\title{
Flexible active matrix addressed displays manufactured by printing and coating techniques
}

\author{
Jun Kawahara, Peter Andersson Ersman, David Nilsson, Kazuya Katoh, Yasukazu Nakata, \\ Mats Sandberg, Marie Nilsson, Goran Gustafsson and Magnus Berggren
}

\section{Linköping University Post Print}

\section{Tweet}

N.B.: When citing this work, cite the original article.

This is the pre-reviewed version of the following article:

Jun Kawahara, Peter Andersson Ersman, David Nilsson, Kazuya Katoh, Yasukazu Nakata, Mats Sandberg, Marie Nilsson, Goran Gustafsson and Magnus Berggren, Flexible active matrix addressed displays manufactured by printing and coating techniques, 2013, Journal of Polymer Science Part B: Polymer Physics, (51), 4, 265-271.

which has been published in final form at:

http://dx.doi.org/10.1002/polb.23213

Copyright: Wiley-Blackwell http://eu.wiley.com/WileyCDA/Brand/id-35.html

Postprint available at: Linköping University Electronic Press http://urn.kb.se/resolve?urn=urn:nbn:se:liu:diva- 88353 
Flexible active matrix addressed displays manufactured by printing and coating techniques

Jun Kawahara, ${ }^{1,2,3}$ Peter Andersson Ersman, ${ }^{1}$ David Nilsson, ${ }^{1}$ Kazuya Katoh, ${ }^{2}$ Yasukazu Nakata, ${ }^{2}$ Mats Sandberg, ${ }^{1}$ Marie Nilsson, ${ }^{1}$ Göran Gustafsson, ${ }^{1}$ Magnus Berggren ${ }^{3}$

${ }^{1}$ Printed Electronics, Acreo AB, Bredgatan 34, SE-60221, Norrköping, Sweden

${ }^{2}$ R\&D Strategy Dept., Lintec Corporation, Warabi, Saitama, 3350005, Japan

${ }^{3}$ Department of Science and Technology, Linköping University, Bredgatan 33, SE-60174, Norrköping, Sweden

Correspondence to: Dr. Peter Andersson Ersman (E-mail: peter.andersson.ersman@acreo.se) Additional Supporting Information may be found in the online version of this article.

\section{ABSTRACT}

A flexible electrochromic active matrix addressed display, including $8 \times 8$ pixels, is demonstrated by using solution processing based on standard printing and coating manufacturing techniques. Each organic electrochromic display (OECD) pixel and its corresponding organic electrochemical transistor (OECT) are located on different sides of the flexible PET substrate. Electronic vias generated through the plastic substrate connects each OECD pixel with one addressing OECT. When comparing this display with actively addressed OECDs with all its components located on the same side, the present approach based on this electronic via-substrate provides an enhanced pixel resolution and a relatively more simplified manufacturing process.

\section{KEYWORDS}

Electrochromic, flexible electronics, organic electronics, printed electronics, matrix display 


\section{INTRODUCTION}

During the last decades an array of different organic electronic devices have been demonstrated and several of them, such as the organic light emitting diodes and field effect transistors, are currently being explored in various applications. ${ }^{1-3}$ These devices are potentially possible to manufacture onto flexible substrates, such as paper and plastics, using common coating and printing tools, thus promising for organic electronics in various distributed and lowcost applications. All-printed organic electronic systems will become a successful generic technology in the future only if devices and systems are robust and simple to manufacture, can operate at low voltages and also if the included electro-active materials are stable and processable from environmentally friendly solvents. Further, traditional large area and high speed printing and coating processes of thin films and patterns are also typically associated with short drying times and moderate annealing temperatures. ${ }^{4}$ Major research efforts have been devoted to establish printing manufacturing protocols for organic electronic components, where perhaps flexible displays represent one of the prime targets. The flexible displays, serving as one among several different communication interfaces, will be integrated with transistor circuits, memories, sensors, batteries, etc. to enable novel electronic labels, e-papers and single-use sensors for applications such as packaging, trace-and-track, safety and diagnostics.

Electrochromic (EC) polymer materials are electrochemically active plastics that reversibly can alter its color upon switching their oxidation state and these materials have been synthesized and characterized by many different groups during the last decades. ${ }^{5-7}$ The EC materials represent one important class of electronic materials suitable for all-organic flexible displays 
and smart windows that are driven at low voltages ( $1 \mathrm{~V}$ ) in either the transmission or reflective mode of operation. ${ }^{3,8,9}$ The color purity, switch time and the color switch contrast are crucial parameters to consider while developing EC materials in display applications. Several recent publications have reported enhanced color switch contrasts as well as multicolor functionality of EC materials. ${ }^{10-17}$ Further, several research groups have demonstrated EC cells incorporated in both actively and passively matrix-addressed cross-point systems, aiming at display systems for e-posters, e-sensor tags, etc. ${ }^{3,8,18}$

Poly(3,4-ethylenedioxythiophene) (PEDOT) doped with poly(styrene sulfonate acid) (PSS), Figure 1 , is one of the most commonly used conducting polymer materials in flexible electronics. Besides exhibiting modulation in its electronic conductivity upon electrochemical switching, PEDOT is also electrochromic. These twinned features provide a multifunctional material that can be employed both as the color switch material in pixels and as the channel and electrode material in transistors, and also as the electrical wires to distribute signals along the cross-point matrix. The oxidation state of PEDOT:PSS is modulated according to:

PEDOT $^{+} \mathrm{PSS}^{-}+\mathrm{M}^{+}+\mathrm{e}^{-} \leftrightarrow \mathrm{PEDOT}^{0}+\mathrm{PSS}^{-} \mathrm{M}^{+}$

$\mathrm{PEDOT}^{+}$denotes the oxidized, optically transparent/light blue and electrically conducting state. $\mathrm{PEDOT}^{0}$ denotes the reduced, dark blue-colored and semiconducting state. Hence, the change in electrical conductivity upon electrochemical switching is utilized in the OECTs, while the modulation in optical absorption is used in the OECD cells. Further, a common solid electrolyte, made from an ionic liquid, a polyelectrolyte or a polymer electrolyte, can serve as the ionic conductor in both the OECTs and the OECD cells. The versatility of PEDOT:PSS, and also that of the electrolyte, result in a matrix-addressed display technology requiring a low number of 
different materials. This then promises for a manufacturing protocol that includes only a few different material deposition and patterning steps. The OECD color switch contrast of PEDOT:PSS alone is rather limited. The EC switch contrast can be further improved by incorporating additional electrochromic materials, such as polyaniline, either on top of the actual PEDOT:PSS display electrode or in the counter electrode. ${ }^{19}$ However, such double/multilayer OECD typically requires a relatively more complex voltage driving scheme to assure reversible and complete switching of the display pixels in between different color states. Further, display cells including multiple EC materials typically exhibit relatively slower switching times, in part explained by a relatively lower electronic conductivity of the additional EC material as compared to that of PEDOT:PSS.

One of the most critical parameters of a matrix-addressed display is the aperture ratio, i.e. the area ratio of the active pixel area vs. the total display area, hereinafter called the fill factor. Hence, one of the prime targets is to always enhance the fill factor, and thereby the overall display appearance, by minimizing the gap between neighboring OECD pixels. In previously reported matrix-addressed displays printed on plastic and paper substrates, respectively, the addressing OECTs and the OECD pixels are located adjacently with respect to each other on the same side of the substrate. ${ }^{3,8}$ This limits the highest achievable fill factor since display cells, transistors and conductors compete against each other to occupy the area of the display surface. One route to enhance the fill factor would be to manufacture only the OECD pixel matrix on the front side of the substrate and then hiding the addressing OECTs and as many of the conducting lines as possible on the back side. Such solution must be accompanied with 
electronic via connections that guide addressing signals from the back side including addressing conductors and all the OECTs to the OECD pixel matrix distributed along the display front side. ${ }^{20}$ In this paper, active matrix addressed organic electrochromic displays (AMOECD), based on an electronic via substrate technology with an enhanced pixel resolution together with a simplified manufacturing process is reported. First, the electrical switching characteristics of OECTs alone are reported. Then, the color switch performance of OECTs combined with OECD pixels, forming the EC smart pixel, is given and finally an AMOECD including $64(8 \times 8)$ EC smart pixels manufactured on a plastic electronic via substrate is presented.

\section{EXPERIMENTAL}

\section{Materials and equipments}

A 50- $\mu \mathrm{m}$ thick polyethylene terephthalate (PET) foil (Melinex 454, purchased from DuPont Teijin) is chosen as the substrate. An electrically conducting carbon screen printing paste (7102 from DuPont, diluted by 20 wt-\% Thinner 3610 from DuPont) serves as the electronically conducting material inside and through the via holes. The PEDOT:PSS dispersion (ICP-1010 purchased from AGFA) is mixed with diethyleneglycol (Sigma-Aldrich) and the surfactant Zonyl FS-300 (DuPont) into a volume composition of 1000:50:1. ${ }^{22}$ The Clevios P Jet HC PEDOT:PSS ink (Heraeus) is used as the formulation in the inkjet process. A water-based polyelectrolyte is prepared by mixing poly(sodium(quaternary ammonium)), see Figure 1, and $10 \mathrm{wt}-\% \mathrm{TiO}_{2}$ powder (KRONOS 2300 purchased from KRONOS). A hydrophobic ink for inkjet printing was prepared by dissolving polystyrene in toluene. The U5603 ink (SunTronic) is used for inkjet printing of silver and a UV curable epoxy resin (SU-8 provided by Microchem) is used as a 
protecting layer. PET films precoated with PEDOT:PSS (Orgacon EL-350 provided by AGFA) function as the gate electrodes. Inkjet printing is performed using a Dimatix DMP-2800, in which the cartridge includes 16 nozzles, each with a drop volume of $10 \mathrm{pl}$.

\section{Device preparation}

Via holes $(8 \times 8)$ are generated through a plastic substrate, Foil 2 in Figure 4 , at a pitch of $1 \mathrm{~mm}$, using a $\mathrm{CO}_{2}$ gas laser-drilling machine (YB-HCSO3, Panasonic Welding Systems Co. Ltd.) operated at a frequency of $3 \mathrm{kHz}$ width the pulse widths of $50 \mu$ s (first pulse) and $25 \mu \mathrm{s}$ (second pulse). This laser drilling process results in via holes of a diameter of about $30 \mu \mathrm{m}$ on the side where the photons enters the PET foil, while the diameters reaches approximately $70 \mu \mathrm{m}$ on the side where the photons escapes the foil. All via holes are then filled with a conducting carbon paste, which is dried for $4-5$ minutes at $120^{\circ} \mathrm{C}$ after the manual printing process.

A $100 \times 500 \mu \mathrm{m}^{2}$ rectangle of PEDOT:PSS, representing the transistor channel, is then inkjetprinted on top of each electronic via on the back side of Foil 2 . The channel is positioned so that each electronic via is located at one of the edges of the PEDOT:PSS rectangle and a total of 64 rectangles are printed for each $8 \times 8$ AMOECD device. The opposite edge of each one of the PEDOT:PSS rectangles is then connected column-wise by inkjet printed silver lines. On top of the OECT channel, the electronic vias are capped by a UV-curable epoxy resin layer and the electrolyte is then deposited on top of each OECT channel. The purpose of the epoxy cap is to prevent electrolyte migration into and through the electronic vias. An electrolyte drop is deposited on top of each via hole and then cured into a semi-solid state by thermal treatment for 1 minute at $60^{\circ} \mathrm{C}$. 
The counter electrodes of the OECD pixels are produced on the front side of Foil 2. First, a grid pattern is formed along this front side by inkjet printing $75 \mu \mathrm{m}$ wide lines from a polystyrene solution. This produces a hydrophobic boundary pattern, including $8 \times 8$ open squares, separated by $2 \mathrm{~mm}$ pitch with respect to each other. Careful registration assures that each via hole is located in the center of each square. Then, a layer of PEDOT:PSS and also a layer of the white opaque electrolyte are subsequently coated along the front side using a wire bar. The difference in surface energy between the plastic substrate and the inkjet printed polystyrene grid promotes both layers to coat only the area inside the polystyrene square grid. The PEDOT:PSS pattern layers ( $500 \mathrm{~nm}$ of thickness) are thermally dried at $110{ }^{\circ} \mathrm{C}$ for $4-5$ minutes and the subsequently deposited electrolyte is annealed at $60^{\circ} \mathrm{C}$ for about 1 minute to form a semi-solidified thick film (20-30 $\mu$ m of thickness).

The pixel electrodes are prepared by uniformly depositing PEDOT:PSS using a wire bar onto Foil 1. A polystyrene grid is then inkjet printed on top of Foil 1 such that the subsequently deposited electrolyte self-aligns into 64 isolated electrolyte squares, where each square of electrolyte defines the respective OECD pixel area.

The gate electrodes consists of a uniformly coated PEDOT:PSS layer on top of PET foil, Orgacon EL-350, which is patterned into 8 parallel rows, each one having the dimension of $2 \times 50 \mathrm{~mm}^{2}$, by using a knife plotter, see Foil 3 in Figure 4. A polystyrene grid pattern is then inkjet printed on top of the sheet of gate electrodes, onto which $\sim 2 \times 2 \mathrm{~mm}^{2}$ electrolyte squares could be surface energy patterned. Finally, the $8 \times 8$ AMOECD is completed by that all three foils are laminated into one vertical stack. 


\section{Device characterization}

Before operating the complete $8 \times 8$ AMOECD display, the electrical performance of individual OECTs, OECD pixels and EC smart pixels were independently characterized using a HP/Agilent 4155 B semiconductor parameter analyzer for the transistor sweeps, and two Keithley 2400 sourcemeter units for the transistor switching time measurements. LabView (National Instruments) graphical user interfaces were used to control the measurements and for data recording and storage.

Digital integrated circuits (ICS) are used to obtain proper addressing of the resulting $8 \times 8$ AMOECD, by supplying parallel voltage outputs from a shift register along the columns responsible for the EC pixel update and by performing row-by-row scanning of the gate voltages from a demultiplexer circuit. However, since digital ICs typically exhibit high slew rate and a static output voltage level defined by the supply voltage, it is advantageous to use an analog circuit in which both slew rate and output voltage levels are tunable. The lifetime of the OECT, and thereby the complete AMOECD system, can be considerably prolonged by lowering the slew rate of the gate voltage bias down to, for example, $1 \mathrm{~V} / 50 \mathrm{~ms}$. Further, the drive protocol of the AMOECD can be optimized if an analog voltage driver is chosen, e.g. such that the amplitudes of the pixel voltage and the gate voltage can be decoupled from each other. We found that $1.5 \mathrm{~V}$ and $2.5 \mathrm{~V}$ are optimal bias voltage amplitudes for the pixel and the gate bias of the AMOECD, respectively. Approximately $3 \mathrm{~s}$ is required in order to complete one $8 \times 8$ AMOECD update cycle, which indicates a total row-by-row scanning rate of about $400 \mathrm{~ms} / \mathrm{row}$. $100 \mathrm{~ms}$ is dedicated for switching the OECT from its off-state to its on-state, $200 \mathrm{~ms}$ is necessary 
to update the EC pixel and finally $100 \mathrm{~ms}$ is needed for the on-to-off switching (closing) of the OECT channel.

\section{RESULTS}

\section{OECT characterization}

First, the current-voltage $(I-V)$ characteristics of the OECT were measured, in which the drain electrode and the transistor channel of the device are electrically connected through the electronic via hole. The on/off switching times and the on/off levels of the drain-source current $\left(I_{D S}\right)$, upon applying a varying gate voltage $\left(V_{G}\right)$ and a constant voltage between the drain and source electrodes $\left(V_{D S}\right)$, were measured on standalone OECTs. The $I_{D S}$ established through the OECT channel was switched off and on in less than $100 \mathrm{~ms}$ and $200 \mathrm{~ms}$, respectively, see Figure 2. The corresponding $I_{D S}$ on-state current is approximately $500 \mu \mathrm{A}$ while the off-state current reaches below $100 \mathrm{nA}$; hence the on/off ratio exceeds 5000 . The relatively slower off-to-on switch is due to that a reduction front propagates outside the electrolyte-PEDOT:PSS OECT channel area into, and also through, the PEDOT:PSS drain electrode, which occurs as the transistor is switched off and as $V_{D S}$ is negative. This occurs since the PSS phase provides enough ion conductivity that enables a reduction front to migrate towards the negatively biased drain electrode. ${ }^{21}$ Re-oxidizing this reduced area, formed between the channel edge and the reduction front within the PEDOT:PSS film, thus limits the speed of the transistor, see Figure 3. The graph shown in Figure 2 only shows slightly longer off-to-on switching time (< 200 ms) as compared to the on-to-off switching time $(<100 \mathrm{~ms})$. However, the off-to-on switch time becomes significantly longer upon increasing the amplitude of $V_{G}$ or if $V_{G}$ is applied for a time period of several ten seconds (data not shown). The combination of the reduction front issue 
and the fact that the PEDOT:PSS-based OECT operates in the depletion mode is a challenge for the overall operation of the resulting AMOECD. One plausible solution to prevent such prolonged switching time, due to propagation of the reduction front, is to deposit a conducting and electrochemically stable material at the drain electrode, that is in direct contact with the PEDOT:PSS channel.

\section{OECT-OECD smart pixel characterization}

The combination of an OECT and an OECD pixel results in an EC smart pixel circuit, combining addressability and pixel functionality, see Figure 4. Here, the smart pixel is manufactured using a substrate with electronic vias, where the OECT is located on the backside of the substrate. The drain electrode of the OECT extends through the electronic via to become the counter electrode of the OECD pixel on the front side of the foil substrate. The $1 \times 1 \mathrm{~mm}^{2}$ smart pixel is completed by laminating Foil 1, Foil 2 and Foil 3 together, see Figure 4. Two pairs of electrolyte layers ensure good ionic contact between the gate electrode and the transistor channel of the OECT and between the pixel and the counter electrodes of the OECD pixel, respectively. The graph in Figure 5 shows the switching characteristics of the EC smart pixel. The updating current $\left(I_{P}\right)$ of the EC smart pixel is supplied by applying a potential difference to the pixel and counter electrode $\left(V_{P}\right)$. The conduction state of the OECT channel, which is dictated by $V_{G}$, controls the level of $I_{P}$. Positive and negative $I_{P}$ values represent coloration and de-coloration of the OECD pixel, respectively. The overall impedance properties of the PEDOT:PSS-based OECD pixel results in a rapid updating current peak, which typically lasts for less than $1 \mathrm{~s}$, during each switching event. The updating voltage protocol and resulting current characteristics of the 
smart pixel promise for updating of individual pixels in the AMOECD device without any significant cross-talk in between neighboring pixels. The actual updating sequence is described in Figure 5.

\section{AMOECD characterization}

An AMOECD demonstrator containing $8 \times 8$ EC smart pixels has been manufactured, that is, a display consisting of 64 OECTs and 64 OECD pixels that all were fully functional. Figure 6 provides the layout scheme of the electronic circuitry of the AMOECD containing only $2 \times 2$ EC smart pixels, where the aim is to explain how the voltages are applied when one of the four OECD pixels is switched. To simplify the manufacturing of this particular AMOECD system, the pixel dimension was enlarged to $\sim 2 \times 2 \mathrm{~mm}^{2}$ with a $2 \mathrm{~mm}$ pitch separation between neighboring OECD pixels. Further, the fill factor of the $8 \times 8$ AMOECD was estimated to $60-70 \%$, which is about the same fill factor as compared to the previously reported AMOECD including considerably larger display pixels manufactured on plastic substrate. ${ }^{3}$ In fact, the pixel area of the present AMOECD display is only $4 \%$ of the pixel area of the previously reported display manufactured on plastics, i.e. $2 \times 2 \mathrm{~mm}^{2}$ vs. $10 \times 10 \mathrm{~mm}^{2}$. In the case that all pixels, transistors and conducting lines are manufactured onto the same side of the substrate and that a fill factor of $60-70 \%$ is targeted, much more advanced printing techniques including improved registration capability would thus be required in order to shrink the transistor and conductor lines down to $4 \%$ of their present dimensions. In this work we have thus proven that the pixel resolution can be considerably increased at the same time as a fill factor of $60-70 \%$ is maintained. This has been possible thanks to that the OECTs and the column lines are defined 
on the back side of Foil 2 and then connected with the OECD pixels through the electronic via substrate. To prove the successful functionality of the reported display the letter " $\mathrm{X}$ " was written into the $8 \times 8$ AMOECD system by addressing the addressing rows and columns along the periphery contacts of the display. Proper updating was achieved without any significant crosstalk in between neighboring pixels in a relatively short amount of time ( $3 \mathrm{~s})$, see Figure 7.

\section{CONCLUSIONS}

An active matrix addressed display consisting of $8 \times 8$ individual electrochromic smart pixels has been manufactured from solution-processable materials on top of a flexible polyester substrate by using various printing and coating techniques. Each smart pixel configuration is based on the combination of an electrochromic pixel and an electrochemical transistor, and the manufacturing is simplified by that both components are relying on the same electrolyte as well as the same electrically conducting and electrochemically active polymer. In addition to this, the major contribution reported is that the electrochromic pixel and its corresponding electrochemical transistor are located on different sides of the flexible plastic substrate, and an electrical connection between the two subcomponents are formed by a via conductor. The electronic via substrate therefore enables enhanced pixel resolution at maintained fill factor of $60-70 \%$ and a simplified manufacturing process of the matrix-addressed display, as compared to the case of a printed display manufactured entirely by an additive printing process on only one side of the substrate. 


\section{ACKNOWLEDGEMENTS}

Prime funding for this project was provided by Lintec Corporation. Also, the authors wish to thank VINNOVA (Printed Electronics Arena), KAW (Power Papers) and Önnesjö Foundation for additional financial support.

\section{REFERENCES AND NOTES}

1. A NanoMarkets whitepaper, "Thin-Film and Printed Batteries: On-Board Solutions for Low-Power Electronics", March 2008.

2. Krebs, F. C.; Fyenbo, J.; Jørgensen, M. J. Mater. Chem. 2010, 20, 8994-9001.

3. Andersson, P.; Forchheimer, R.; Tehrani, P.; Berggren, M. Adv. Funct. Mater. 2007, 17, 3074-3082.

4. Berggren, M.; Nilsson, D; Robinson, N, D. Nat. Mater. 2007, 6, 3-5.

5. Groenendaal, L.; Jonas, F.; Freitag, D.; Pielartzik, H.; Reynolds, J. R. Adv. Mater. 2000, 12, 7, 481-494.

6. Hellström, S.; Henriksson, P.; Kroon, R.; Wang, E.; Andersson, M. R. Org. Electron. 2011, 12, 8, 14061413.

7. Apaydin, D. H.; Akpinar, H.; Sendur, M.; Toppare, L. J. Electroanal. Chem. 2012, 665, 52-57.

8. Andersson, P.; Nilsson, D.; Svensson, P.-O.; Chen, M.; Malmström, A.; T. Remonen, T.; Kugler, T.;

Berggren, M. Adv. Mater. 2002, 14, 20, 1460-1464.

9. Prakash, R.; Santhanam, K. S. V. J. Solid State Electrochem. 1998, 2, 123-125.

10. Mortimer, R. J.; Dyer, A.L.; Reynolds, J. R. Displays 2006, 27, 2-18.

11. Gunbas, G. E.; Durmus, A.; Toppare, L. Adv. Funct. Mater. 2008, 18, 2026-2030.

12. Shi, P.; Amb, C. M.; Knott, E. P.; Thompson, E. J.; Liu, D. Y.; Mei, J.; Dyer, A. L.; Reynolds, J. R. Adv. Mater. 2010, 22, 4949-4953.

13. Ikeda, T.; Higuchi, M.; Kruth, D. G. J. Am. Chem. Soc. 2009, 131, 9158-9159.

14. Patra, A.; Bendikov, M. J. Mater. Chem. 2010, 20, 422-433.

15. Maier, A.; Rabindranath, R.; Tieke, B. Adv. Mater. 2009, 21, 959-963.

16. Kawahara, J.; Andersson Ersman, P.; Engquist, I.; Berggren, M. Org. Electron. 2012, 13, 469-474.

17. Beaujuge, P. M.; Ellinger, S.; Reynolds, J. R. Nat Mater. 2008, 7, 10, 795-799.

18. Kobayashi, N.; Miura, S.; Nishimura, M.; Urano, H. Sol. Energy Mater. Sol. Cells, 2008, 92, 136-139.

19. Jang, J. E.; Cha, S. N.; Lee, J. M.; Kim, J. J.; Amaratunga, G. A. J.; Jung, J. E. Opt. Lett. 2012, 37, 235-237.

20. Argun, A. A.; Berard, M.; Aubert, P.-H.; Reynolds, J. R. Adv. Mater. 2005, 17, 4, 422-426.

21. Johansson, T.; Persson, N.-K.; Inganäs, O. J. Electrochem. Soc. 2004, 151, E119-E124.

22. Crispin, X.; Jakobsson, F. L. E.; Crispin, A.; Grim, P. C. M.; Andersson, P.; Volodin, A.; van Haesendonck, C.; van der Auweraer, M.; Salaneck, W. R.; Berggren, M. Chem. Mater. 2006, 18, 4354-4360. 
PEDOT<smiles>CC(C)(C)c1sc(C(F)(F)F)c2c1OCCO2</smiles>

PSS<smiles>CCC(CC)c1cccc(S(=O)(=O)O)c1</smiles>

Polyelectrolyte

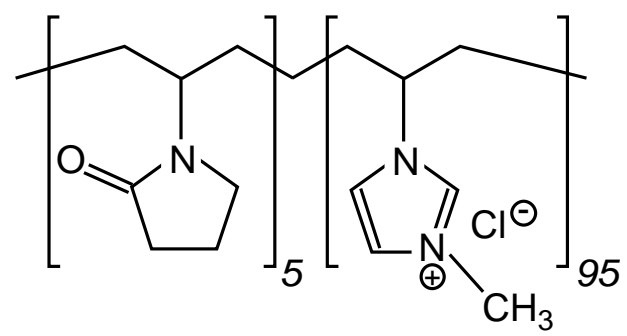

FIGURE 1 The chemical structures of the materials used in the reported devices and display system; the conducting electrochromic polymer PEDOT (left), its counter polyanion PSS (middle) and the polyelectrolyte poly(sodium(quaternary ammonium)) (right).
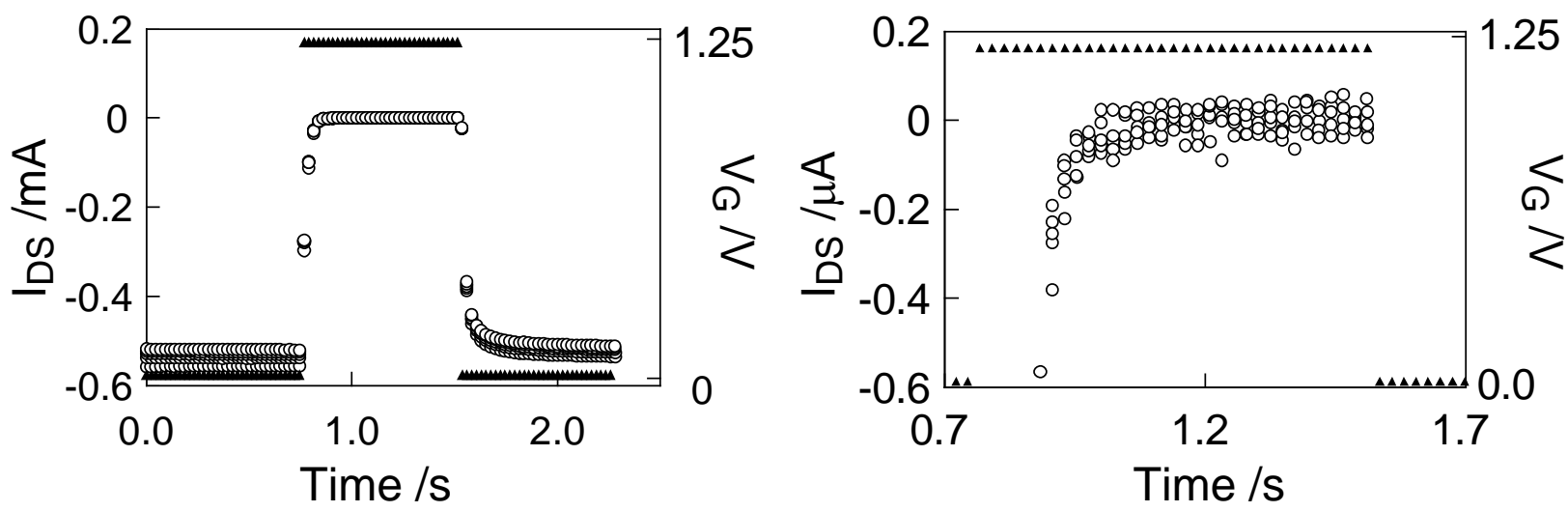

FIGURE 2 The $I_{D S}$ (open circles) behavior of the OECT while switching $V_{G}$ (filled triangles) between 0 and $1.25 \mathrm{~V} . V_{D S}=-1 \mathrm{~V}$, the time between two consecutive data points is $20 \mathrm{~ms}$ and 5 sweeps are plotted on top of each other. Right: magnification of the same data to show that the off-current levels are lower than 50 nA. 


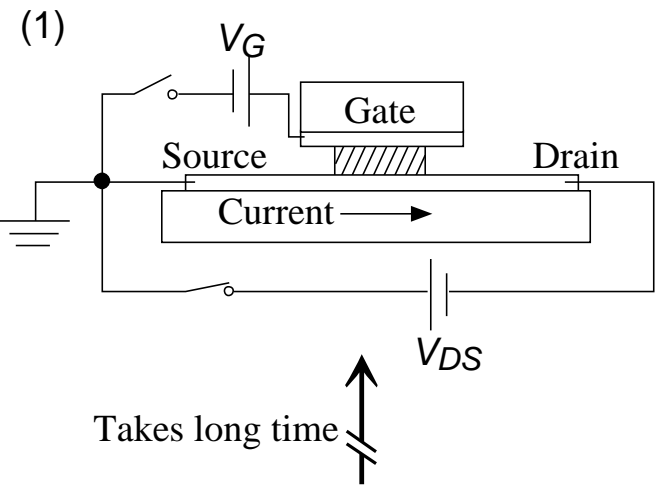

(4)

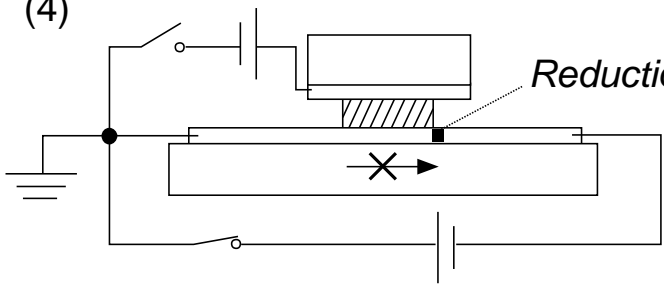

(2)

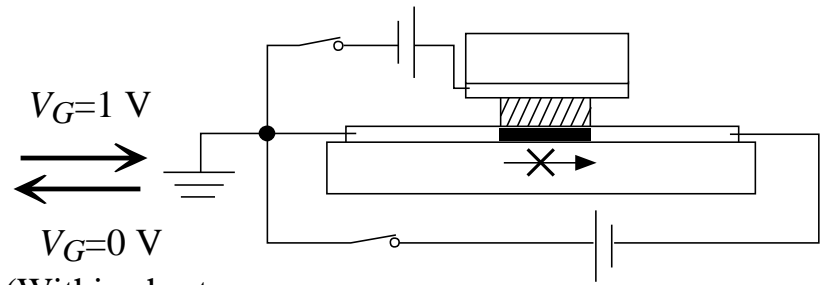

(Within short enough time)
After long time with $V_{G}=1 \mathrm{~V}$

(3)
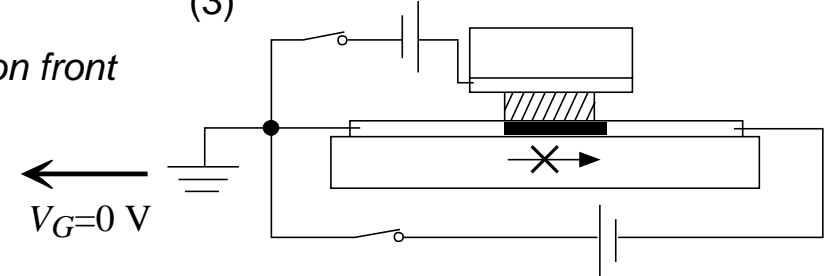

FIGURE 3 A cross-sectional image explaining the reduction front that prolongs the switching time of the OECT. (1) The OECT is in its pristine conducting state when $V_{G}=0 \mathrm{~V}$, which results in that $I_{D S}$ flows through the channel upon applying $V_{D S}$. (2) Upon applying $V_{G}$, the PEDOT:PSS in the OECT channel is electrochemically reduced to its non-conducting state, hence, $I_{D S}$ is switched off. As long as $V_{G}$ is set to 0 $V$ within a sufficiently short amount of time, typically $<500 \mathrm{~ms}$, the time required to switch the OECT channel back to its conducting state is about the same as compared to the opposite switching event. (3) However, by applying $V_{G}$ for longer time results in that the reduced PEDOT:PSS propagates outside the area covered by the electrolyte. (4) Such reduction front prolongs the off-to-on switching event severely upon switching $V_{G}$ to $0 \mathrm{~V}$, which in turn results in non-symmetric OECT switching characteristics. 

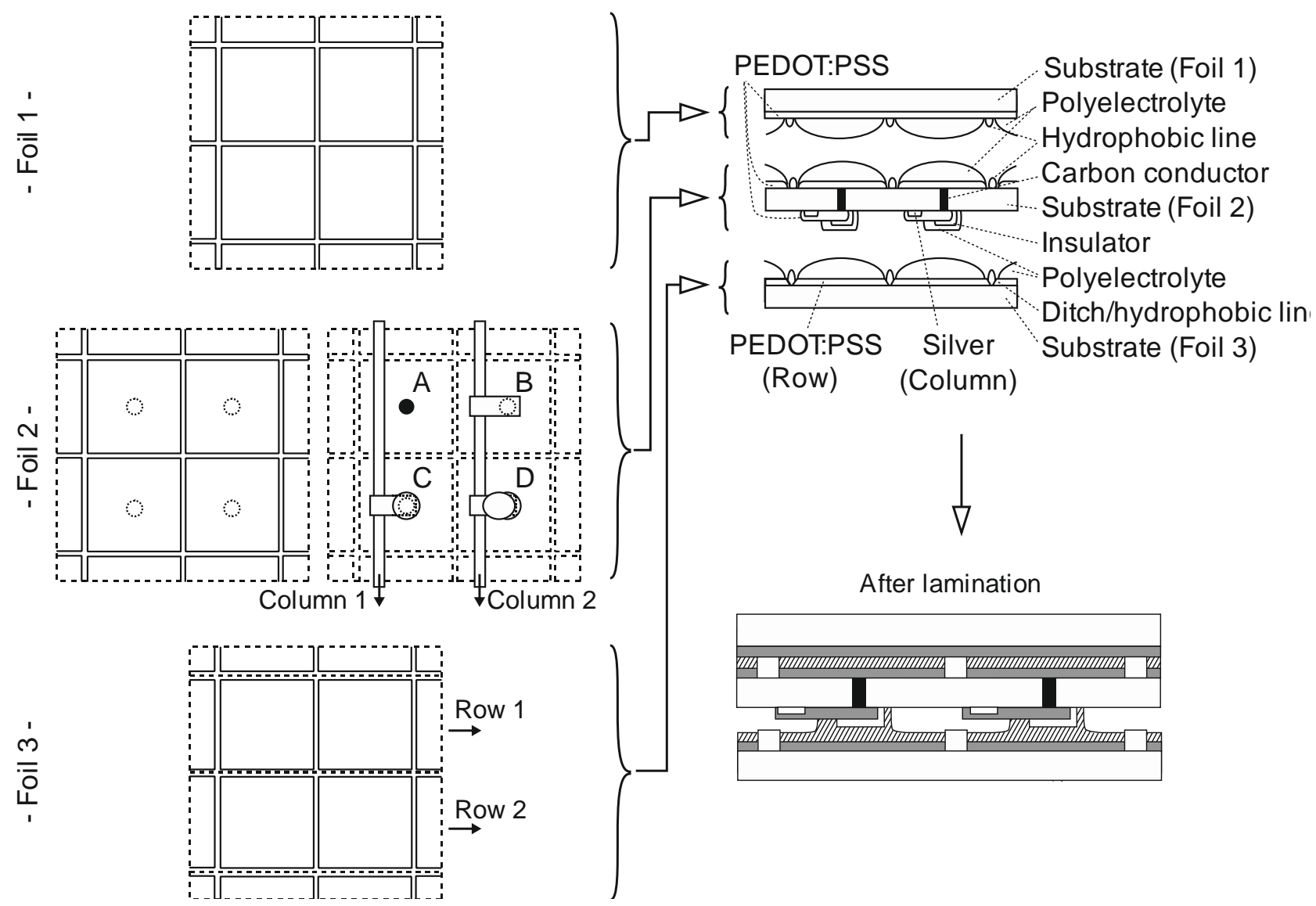

FIGURE 4 Illustrations of the top view (left) and the cross-sectional view (right) of Foil 1, Foil 2 and Foil 3 are here given for a $2 \times 2$ AMOECD architecture. The top view of Foil 2 also indicates the sequences for manufacturing the OECT channel: the electronic via and the column line (A), the OECT channel (B), an insulating layer (C), and the electrolyte layer (D). The lower right illustration shows the resulting AMOECD after lamination of the three foils.

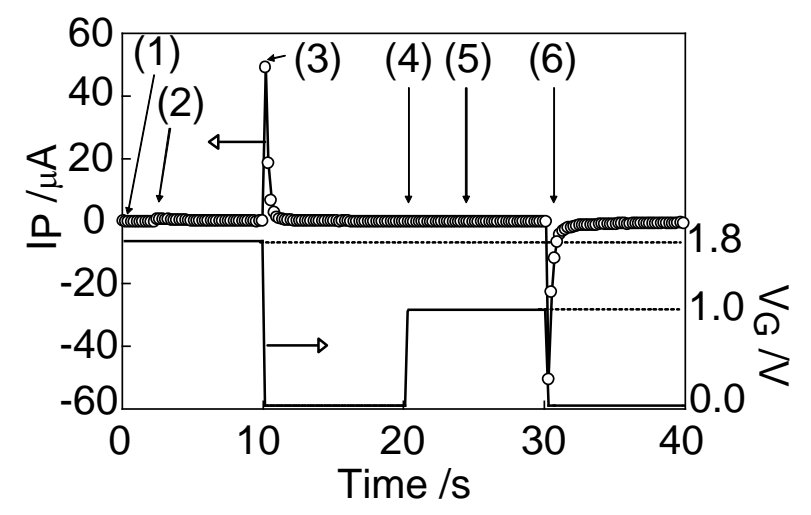

FIGURE 5 The graph shows the functionality test of an EC smart pixel. $I_{P}$ and $V_{G}$ are plotted as a function of time, and the pitch between two consecutive data points is $200 \mathrm{~ms}$. (1) The OECT channel was switched off by applying $V_{G}=1.75 \mathrm{~V}$. (2) The EC pixel voltage, $V_{P}=1.0 \mathrm{~V}$, was applied at $t=3 \mathrm{~s}$, which results in a small leakage current that is incapable of creating cross-talk coloration of the pixel. (3) The OECT channel was switched on $\left(V_{G}=0 \mathrm{~V}\right.$ at $\left.t=10 \mathrm{~s}\right)$, which results in pixel coloration. (4) The OECT 
channel was switched off $\left(V_{G}=1.0 \mathrm{~V}\right.$ at $\left.t=20 \mathrm{~s}\right),(5) V_{P}$ was switched to $0 \mathrm{~V}(t=25 \mathrm{~s})$, and it is shown that the non-conducting channel prevents spontaneous decoloration of the OECD pixel. (6) The pixel is decolored by that the OECT channel was switched on $\left(V_{G}=0 \mathrm{~V}\right.$ at $\left.t=30 \mathrm{~s}\right)$.

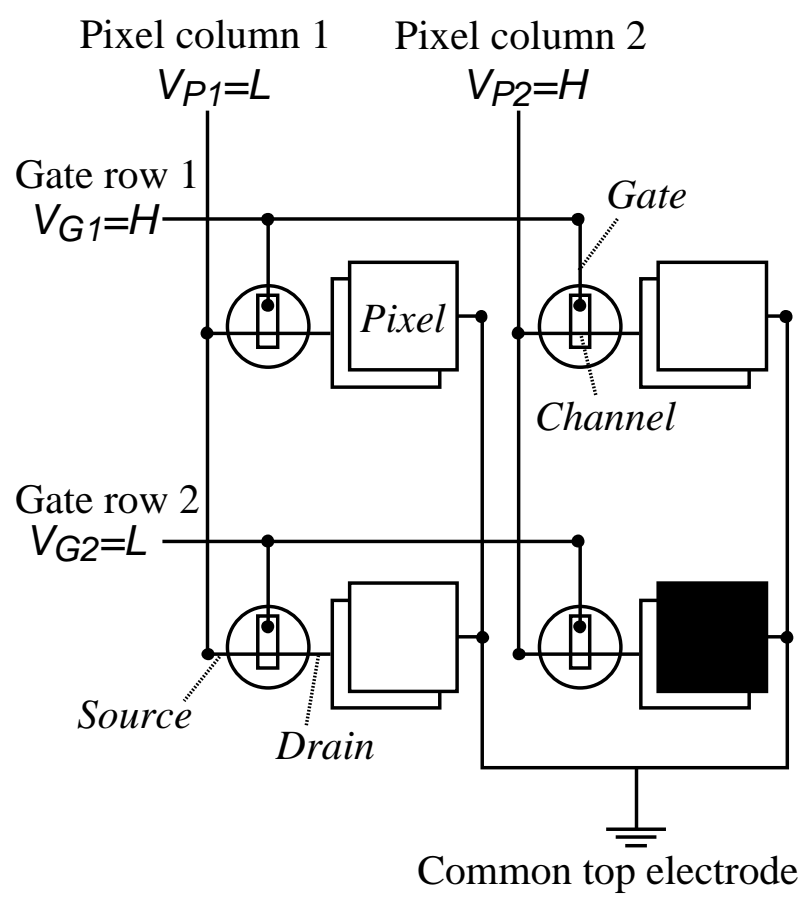

FIGURE 6 A $2 \times 2$ section of the circuit layout of the AMOECD is given. The required applied voltages to update the lower right pixel is given and $L$ and $H$ represents a low and a high digital voltage level, respectively, e.g. $L=0 \mathrm{~V}$ and $H=2 \mathrm{~V}$. The voltage applied to each gate electrode row $\left(V_{G}\right)$ controls the conductivity of all OECTs along that specific row, while the column lines address the source/counter electrodes of all OECTs/OECD pixels along that specific column upon applying the drain-source potential $\left(V_{D S}\right) /$ pixel potential $\left(V_{P}\right)$. The OECD pixel electrodes are represented by the top layer, which electronically is set to common ground. Note that the OECT is operating in depletion mode, hence, the channel is non-conducting when $V_{G}=H$. 


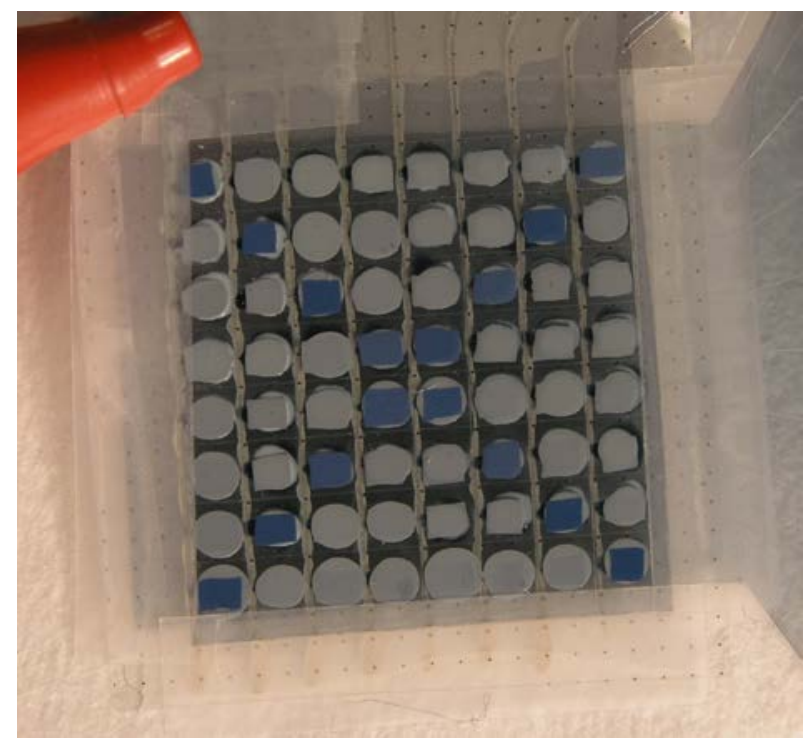

FIGURE 7 A photograph showing the operation of an $8 \times 8$ AMOECD demonstrator in which the letter " $X$ " successfully is written by the external addressing electronics without cross-talk effects. A video clip showing the $\sim 3 \mathrm{~s}$ updating cycles that result in the $8 \times 8$ AMOECD image can be seen in the Supporting Information available online. 